PROCEEDINGS OF THE

AMERICAN MATHEMATICAL SOCIETY

Volume 127, Number 12, Pages 3461-3466

S 0002-9939(99)04898-4

Article electronically published on May 13, 1999

\title{
EXISTENCE OF HOMOGENEOUS IDEALS FITTING INTO LONG BOURBAKI SEQUENCES
}

\author{
MUTSUMI AMASAKI
}

(Communicated by Wolmer V. Vasconcelos)

\begin{abstract}
For any finitely generated torsion-free graded module over a polynomial ring, there exists a homogeneous ideal fitting into an exact sequence similar to a Bourbaki sequence even though its height is not restricted to two.
\end{abstract}

\section{INTRODUCTION}

Given a homogeneous ideal $I$ of height $p \geq 2$ in a polynomial ring $R:=$ $k\left[x_{1}, \ldots, x_{r}\right]$, there is a finitely generated torsion-free graded $R$-module $M$ with no free direct summand satisfying $\operatorname{Ext}_{R}^{i}(M, R)=0$ for $i=1, \ldots, p-1$ that fits into an exact sequence of the form

$$
0 \longrightarrow S_{p-1} \longrightarrow S_{p-2} \longrightarrow \cdots \longrightarrow S_{1} \longrightarrow S_{0} \oplus M \longrightarrow I(c) \longrightarrow 0,
$$

where $c$ is an integer and $S_{i}(0 \leq i \leq p-1)$ are finitely generated graded free $R$-modules (see e.g. [6, Section 1], [12, Section 1]). By this sequence one obtains

$$
H_{\mathfrak{m}}^{i-1}(R / I)(c) \cong H_{\mathfrak{m}}^{i}(M) \quad \text { for } \quad i=1, \ldots, \operatorname{dim}(R / I)=r-p .
$$

Since $H_{\mathfrak{m}}^{i}(M)=0$ for $i=r-p+1, \ldots, r-1$ and $i=0$ by local duality, considering the local cohomologies of $R / I$ is the same thing as considering those of $M$.

Keeping the above observation in mind we are interested in the following problem. First, fix an integer $p \geq 2$ and a finitely generated torsion-free graded $R$-module $M$ with no free direct summand satisfying $\operatorname{Ext}_{R}^{i}(M, R)=0$ for $i=$ $1, \ldots, p-1$. Next, let $\Im(M, p)$ be the set of all homogeneous ideals $I$ in $R$ of height $p$ fitting into exact sequences of the form $(*)$. With this notation, describe all the members of $\mathfrak{I}(M, p)$ in full generality.

Perhaps the most popular way to study the structure of $\mathfrak{I}(M, p)$ is to do so in the framework of even linkage theory (see $[8,11,12,14])$. But we want to propose another approach based on the analysis of basic sequences. Roughly speaking, the basic sequence $B_{R}(I)=\left(\bar{n}^{1} ; \bar{n}^{2} ; \cdots ; \bar{n}^{r+1}\right)$ of a homogeneous ideal $I$ is a sequence of integers obtained by arranging in a suitable order the degrees of the Gröbner basis of $I$ with respect to generic coordinates, where $\bar{n}^{i}$ denotes a subsequence for each $i$ (see [3, Section 1], [5, Example 4.1]). A similar sequence can also be defined for an arbitrary finitely generated graded $R$-module (see [5, Section 2]) and, if $I \in \mathfrak{I}(M, p)$

Received by the editors September 26, 1997 and, in revised form, February 10, 1998. 1991 Mathematics Subject Classification. Primary 13D02; Secondary 13D03.

(C)1999 American Mathematical Society 
with $B_{R}(I)=\left(\bar{n}^{1} ; \bar{n}^{2} ; \cdots ; \bar{n}^{r+1}\right), B_{R}(M)=\left(\bar{\nu}^{1} ; \bar{\nu}^{2} ; \cdots ; \bar{\nu}^{r+1}\right)$, then we have

$$
\left\{\begin{aligned}
\bar{n}^{p} & =\left(\bar{w}^{\prime}, \bar{\nu}^{p}+c\right) \quad \text { up to permutation and } \\
\bar{n}^{i} & =\bar{\nu}^{i}+c \text { for } i=p+1, \ldots, r+1
\end{aligned}\right.
$$

with a suitable sequence of integers $\bar{w}^{\prime}$, where $\bar{a}+c=\left(a_{1}+c, \ldots, a_{l}+c\right)$ for a sequence $\bar{a}=\left(a_{1}, \ldots, a_{l}\right)$ (see $[6$, Section 2]). This formula, however, is not enough for characterizing all possible basic sequences of the elements of $\mathfrak{I}(M, p)$.

So far we have been successful only in two special cases. In the case $p=2$, the formula $(* *)$ can further be developed to give a complete description of the basic sequences of the elements of $\mathfrak{I}(M, 2)$ (see [7, Section 2]). Our results describe also in a different way the main theorem in the above-mentioned two codimensional even linkage theory. In particular, one can understand the numerical function $\theta_{X}$ defined by Nollet in [13] from a structural point of view (see [7, Theorem 3.3]). When $M$ is Buchsbaum, or equivalently, when the ring $R / I$ is Buchsbaum, the relation $(* *)$, together with the Borel fixedness of generic initial ideals, determines almost completely the basic sequences of the elements of $\mathfrak{I}(M, p)$ (see [1, Sections 2 and 3], [2], [3, Sections 5 and 6]).

Before proceeding further, we have to know first whether the set $\mathfrak{I}(M, p)$ is empty or not. The aim of this paper is to settle this question. In fact we will prove that $\Im(M, p) \neq \emptyset$ for an arbitrary finitely generated torsion-free graded $R$-module $M$ with no free direct summand satisfying $\operatorname{Ext}_{R}^{i}(M, R)=0$ for $i=1, \ldots, p-1$. To the best of our knowledge, there seems no published proof of this fact except for the case $p=2$ (see e.g. [9, Chapitre VII, Section 4, Théorème 6]).

\section{MOdUles AND IDEALS VIA COMPLEXES}

Given integers $p \geq 2, s \leq-1$ and a homogeneous ideal $\mathfrak{a} \subset R$ of height larger than or equal to $p$, let $\mathcal{C}(p, s, \mathfrak{a})$ denote the set of complexes $F_{\bullet}$ of finitely generated graded free $R$-modules bounded on both sides satisfying the following conditions:

(i) $H_{i}\left(F_{\bullet}\right)=0$ for $i \geq 0$,

(ii) $F_{s} \neq 0$ and $F_{i}=0$ for $i<s$,

(iii) $\mathfrak{a}^{l} H_{i}\left(F_{\bullet}\right)=0$ for all $i<0$ and $l \gg 0$.

Lemma 1. Let $p \geq 2, s \leq-1$ be integers, a a homogeneous ideal in $R$ of height larger than or equal to $p$, and $F_{\bullet}$ an element of $\mathcal{C}(p, s, \mathfrak{a})$.

(1) If $s<-1$, then there exist a homogeneous ideal $\mathfrak{a}^{\prime} \subset \mathfrak{a}$ of height larger than or equal to $p$ and a complex $F_{\bullet}^{\prime} \in \mathcal{C}\left(p, s+1, \mathfrak{a}^{\prime}\right)$ such that $F_{i}^{\prime}=F_{i}$ for $i>p-1$.

(2) If $s=-1$ and $\operatorname{rank}_{R}\left(F_{-1}\right)>1$, then there exist a homogeneous ideal $\mathfrak{a}^{\prime} \subset \mathfrak{a}$ of height $p$ and a complex $F_{\bullet}^{\prime} \in \mathcal{C}\left(p,-1, \mathfrak{a}^{\prime}\right)$ such that $\operatorname{rank}_{R}\left(F_{-1}^{\prime}\right)=\operatorname{rank}_{R}\left(F_{-1}\right)-1$ and $F_{i}^{\prime}=F_{i}$ for $i>p-1$.

(3) If $s=-1$ and $\operatorname{rank}_{R}\left(F_{-1}\right)=1$, then there exist a homogeneous ideal $\mathfrak{a}^{\prime} \subset \mathfrak{a}$ of height $p$ and a complex $F_{\bullet}^{\prime} \in \mathcal{C}\left(p,-1, \mathfrak{a}^{\prime}\right)$ such that $\operatorname{rank}_{R}\left(F_{-1}^{\prime}\right)=1$, $\operatorname{codim}\left(H_{-1}\left(F_{\bullet}^{\prime}\right)\right)=p$, and $F_{i}^{\prime}=F_{i}$ for $i>p-1$.

Proof. We denote by $\varphi_{i}: F_{i} \longrightarrow F_{i-1}(i \in \mathbf{Z})$ the differentials of $F_{\bullet}$. By the ArtinRees lemma, there exists for each $i<0$ an integer $l_{i}$ such that $\mathfrak{a}^{l} F_{i} \cap \operatorname{Ker}\left(\varphi_{i}\right)=$ $\mathfrak{a}^{l-l_{i}}\left(\mathfrak{a}^{l_{i}} F_{i} \cap \operatorname{Ker}\left(\varphi_{i}\right)\right)$ for all $l \geq l_{i}$. Since $\mathfrak{a}^{l} H_{i}\left(F_{\bullet}\right)=0$ for all $i<0$ and $l \gg 0$ and $F_{i}=0$ for $i<s$, there is therefore an integer $\bar{l}$ such that

$$
\mathfrak{a}^{\bar{l}} F_{i} \cap \operatorname{Ker}\left(\varphi_{i}\right) \subset \mathfrak{a}^{\bar{l}-l_{i}} \operatorname{Ker}\left(\varphi_{i}\right) \subset \operatorname{Im}\left(\varphi_{i+1}\right)
$$


for all $i<0$. Since $\operatorname{ht}\left(\mathfrak{a}^{\bar{l}}\right)=\operatorname{ht}(\mathfrak{a}) \geq p$, we can pick $p$ homogeneous elements $f_{1}, \ldots, f_{p} \in \mathfrak{a}^{\bar{l}}$ which form an $R$-regular sequence. Using these elements, let $K_{\bullet}:=$ $K\left(f_{1}, \ldots, f_{p} ; R\right)$ • be the Koszul complex with differential $\partial_{\bullet}$. Note that

$$
H_{i}\left(K_{\bullet}\right)=0 \quad \text { for } \quad i \neq 0 \text { and }\left(f_{1}, \ldots, f_{p}\right) H_{0}\left(K_{\bullet}\right)=0 .
$$

From now on, given a complex $C$. of graded $R$-modules and an integer $n$, we denote by $C_{\bullet}[n]\left(\right.$ resp. $\left.C_{\bullet}(n)\right)$ the complex $C_{\bullet}^{\prime}\left(\right.$ resp. $\left.C_{\bullet}^{\prime \prime}\right)$ such that $C_{i}^{\prime}=C_{i+n}$ $\left(\right.$ resp. $\left.C_{i}^{\prime \prime}=C_{i}(n)\right)$.

(1) For proving the first assertion, we construct a chain map $\mu_{\bullet}: K_{\bullet}[-s] \otimes_{R}$ $F_{s} \longrightarrow F_{\bullet}$ such that $\mu_{s}$ is an isomorphism and then consider its mapping cone. To begin with, let $\mu_{s}: K_{0} \otimes F_{s}=F_{s} \longrightarrow F_{s}$ be the identity mapping and $\mu_{i}:=0$ for $i<s$. Let $j \geq s$ be an integer. If we have already defined homomorphisms $\mu_{i}: K_{i-s} \otimes F_{s} \longrightarrow F_{i}$ for all $i \leq j$ satisfying $\mu_{i-1} \circ\left(\partial_{i-s} \otimes \operatorname{id}_{F_{s}}\right)=\varphi_{i} \circ \mu_{i}$, then, since

$$
\begin{aligned}
\operatorname{Im}\left(\mu_{j} \circ\left(\partial_{j+1-s} \otimes \operatorname{id}_{F_{s}}\right)\right) & \subset\left(f_{1}, \ldots, f_{p}\right) F_{j} \cap \operatorname{Ker}\left(\varphi_{j}\right) \\
& \subset \mathfrak{a}^{\bar{l}} F_{j} \cap \operatorname{Ker}\left(\varphi_{j}\right) \subset \operatorname{Im}\left(\varphi_{j+1}\right)
\end{aligned}
$$

by (1.1) and condition (i), there exists a homomorphism $\mu_{j+1}: K_{j+1-s} \otimes F_{s} \longrightarrow$ $F_{j+1}$ satisfying $\mu_{j} \circ\left(\partial_{j+1-s} \otimes \operatorname{id}_{F_{s}}\right)=\varphi_{j+1} \circ \mu_{j+1}$. Thus, we obtain a desired chain map $\mu_{\bullet}$ inductively. Let $\operatorname{con} \mu_{\bullet}$ be its mapping cone with differential $\lambda_{\bullet}$. Since $K_{i-s}=0$ for $i<s, i \geq p-1$, we have $\operatorname{con}\left(\mu_{\bullet}\right)_{i}=F_{i}$ for $i>p-1$ and $\operatorname{con}\left(\mu_{\bullet}\right)_{i}=0$ for $i<s$. Moreover, it follows from the long exact sequence arising from

$$
0 \longrightarrow F_{\bullet} \longrightarrow \operatorname{con} \mu_{\bullet} \longrightarrow K_{\bullet}[-s-1] \otimes_{R} F_{s} \longrightarrow 0
$$

that $H_{i}\left(\operatorname{con} \mu_{\bullet}\right)=0$ for $i \geq 0$ and that $\left(f_{1}, \ldots, f_{p}\right)^{l} H_{i}\left(\operatorname{con} \mu_{\bullet}\right)=0$ for all $i<0$ and $l \gg 0$. Since $\left.\lambda_{s+1}\right|_{K_{0} \otimes F_{s}}=\mu_{s}$ is an isomorphism, we can cancel out free direct summands $K_{0} \otimes F_{s}$ and $\operatorname{con}\left(\mu_{\bullet}\right)_{s}=F_{s}=\lambda_{s+1}\left(K_{0} \otimes F_{s}\right)$ from $\operatorname{con}\left(\mu_{\bullet}\right)_{s+1}$ and $\operatorname{con}\left(\mu_{\bullet}\right)_{s}$ respectively, to obtain a new complex $F_{\bullet}^{\prime}$ such that $F_{i}^{\prime}=\operatorname{con}\left(\mu_{\bullet}\right)_{i}$ for $i>s+1, F_{s+1}^{\prime}=\operatorname{con}\left(\mu_{\bullet}\right)_{s+1} / K_{0} \otimes F_{s}, F_{i}^{\prime}=0$ for $i<s+1$, and $H_{i}\left(\operatorname{con} \mu_{\bullet}\right) \cong H_{i}\left(F_{\bullet}^{\prime}\right)$ for all $i \in \mathbf{Z}$. Let $\mathfrak{a}^{\prime}:=\left(f_{1}, \ldots, f_{p}\right)$. Since $\operatorname{rank}_{R}\left(F_{s+1}^{\prime}\right)=\operatorname{rank}_{R}\left(F_{s+1}\right)>0$ by conditions (ii) and (iii), we obtain $F_{\bullet}^{\prime} \in \mathcal{C}\left(p, s+1, \mathfrak{a}^{\prime}\right)$ as desired.

(2) In the second case, we construct a chain map $\mu_{\bullet}: K_{\bullet}[2](c) \longrightarrow F_{\bullet}$ with a suitable $c \in \mathbf{Z}$ such that the image of $\mu_{-1}: K_{1}(c) \longrightarrow F_{-1}$ is a free direct summand of $F_{-1}$ of rank two, and then consider its mapping cone. To this end, let $R(-a) \oplus R(-b)$ be a free direct summand of $F_{-1}$ with $a \geq b$, so that $F_{-1}=$ $(R(-a) \oplus R(-b)) \oplus P$ for some graded free $R$-module $P$. By multiplying either $f_{1}$ or $f_{2}$ by a suitable homogeneous polynomial, if necessary, we may assume with no loss of generality that $\operatorname{deg}\left(f_{1}\right)-\operatorname{deg}\left(f_{2}\right)=a-b$. Let $c:=\operatorname{deg}\left(f_{1}\right)-a$. Then, since $K_{1}=\bigoplus_{i=1}^{p} R\left(-\operatorname{deg}\left(f_{i}\right)\right)$, we have $K_{1}(c)=(R(-a) \oplus R(-b)) \oplus Q$ with $Q:=$ $\bigoplus_{i=3}^{p} R\left(c-\operatorname{deg}\left(f_{i}\right)\right)$. Let $\mu_{i}:=0$ for $i<-1$, and let

$$
\mu_{-1}=\left(\begin{array}{cc}
\left(\begin{array}{ll}
1 & 0 \\
0 & 1
\end{array}\right) & \mathrm{O} \\
\mathrm{O} & \mathrm{O}
\end{array}\right) \text {. }
$$

Then, $\operatorname{Im}\left(\mu_{-1} \circ \partial_{2}\right) \subset \mathfrak{a}^{\bar{l}} F_{-1} \subset \operatorname{Im}\left(\varphi_{0}\right)$. There is therefore a homomorphism $\mu_{0}$ : $K_{2}(c) \longrightarrow F_{0}$ such that $\mu_{-1} \circ \partial_{2}=\varphi_{0} \circ \mu_{0}$. Since $H_{i}\left(F_{\bullet}\right)=0$ for $i \geq 0$, we can construct $\mu_{i}(i \geq 0)$ successively so that $\mu_{\bullet}$ becomes a chain map. Let $\operatorname{con} \mu_{\bullet}$ be its mapping cone with differential $\lambda_{\bullet}$. Then $\operatorname{con}\left(\mu_{\bullet}\right)_{i}=F_{i}$ for $i>p-1$ and 
$\operatorname{con}\left(\mu_{\bullet}\right)_{i}=0$ for $i<-1$ since $K_{i+2}=0$ for $i \geq p-1, i<-2$. Moreover it follows from the long exact sequence arising from

$$
0 \longrightarrow F_{\bullet} \longrightarrow \operatorname{con} \mu_{\bullet} \longrightarrow K_{\bullet}[1](c) \longrightarrow 0
$$

that $H_{i}\left(\operatorname{con} \mu_{\bullet}\right)=0$ for $i \geq 0$ and that $\left(f_{1}, \ldots, f_{p}\right)^{l} H_{-1}\left(\operatorname{con} \mu_{\bullet}\right)=0$ for $l \gg 0$. Since $\mu_{-1}$ maps $R(-a) \oplus R(-b) \subset K_{1}(c)$ isomorphically onto $R(-a) \oplus R(-b) \subset$ $F_{-1}$, we can cancel out $R(-a) \oplus R(-b)$ and $\lambda_{0}(R(-a) \oplus R(-b))$ from $\operatorname{con}\left(\mu_{\bullet}\right)_{0}$ and $\operatorname{con}\left(\mu_{\bullet}\right)_{-1}$ respectively, to obtain a complex $F_{\bullet}^{\prime}$ such that $F_{i}^{\prime}=\operatorname{con}\left(\mu_{\bullet}\right)_{i}$ for $i>0, F_{0}^{\prime}=\operatorname{con}\left(\mu_{\bullet}\right)_{0} /(R(-a) \oplus R(-b)), F_{-1}^{\prime}=\operatorname{con}\left(\mu_{\bullet}\right)_{-1} / \lambda_{0}(R(-a) \oplus R(-b)) \cong$ $P \oplus K_{0}(c)$, and $H_{i}\left(\operatorname{con} \mu_{\bullet}\right) \cong H_{i}\left(F_{\bullet}^{\prime}\right)$ for all $i \in \mathbf{Z}$. Let $\mathfrak{a}^{\prime}:=\left(f_{1}, \ldots, f_{p}\right)$. Since $\operatorname{rank}_{R}\left(F_{-1}^{\prime}\right)=\operatorname{rank}_{R}\left(F_{-1}\right)+1-2=\operatorname{rank}_{R}\left(F_{-1}\right)-1$, we get $F_{\bullet}^{\prime} \in \mathcal{C}\left(p,-1, \mathfrak{a}^{\prime}\right)$ as desired.

(3) In the last case, we construct a chain map $\mu_{\bullet}: K_{\bullet}[2](c) \longrightarrow F_{\bullet}$ such that $\mu_{-1}$ is a surjection and then consider its mapping cone. Let $a$ be an integer with $F_{-1}=R(-a)$ and let $c:=\operatorname{deg}\left(f_{1}\right)-a$. Let further $\mu_{i}:=0$ for $i<-1, P:=$ $\bigoplus_{i=2}^{p} R\left(c-\operatorname{deg}\left(f_{i}\right)\right)$, and $\mu_{-1}:=(1,0)$ a homomorphism from $K_{1}(c)=R(-a) \oplus P$ to $F_{-1}=R(-a)$. Then, the $\operatorname{Im}\left(\mu_{-1} \circ \partial_{2}\right)$ is contained in $\operatorname{Im}\left(\varphi_{0}\right)$ as in the preceding case. Hence we can construct $\mu_{i}(i \geq 0)$ successively so that $\mu_{\bullet}$ becomes a chain map. Let $\operatorname{con} \mu_{\bullet}$ be its mapping cone with differential $\lambda_{\bullet}$. Then, again as in the preceding case, we have $\operatorname{con}\left(\mu_{\bullet}\right)_{i}=F_{i}$ for $i>p-1, \operatorname{con}\left(\mu_{\bullet}\right)_{i}=0$ for $i<-1$, $H_{i}\left(\operatorname{con} \mu_{\bullet}\right)=0$ for $i \geq 0$, and moreover, it follows from the exact sequence

$$
0 \longrightarrow H_{-1}\left(F_{\bullet}\right) \longrightarrow H_{-1}\left(\operatorname{con} \mu_{\bullet}\right) \longrightarrow H_{0}\left(K_{\bullet}(c)\right) \longrightarrow 0
$$

that $\left(f_{1}, \ldots, f_{p}\right)^{l} H_{-1}\left(\operatorname{con} \mu_{\bullet}\right)=0$ for all $l \gg 0$ and that $\operatorname{codim}\left(H_{-1}\left(\operatorname{con} \mu_{\bullet}\right)\right)=p$ in $\operatorname{Spec}(R)$. Since $\left.\mu_{-1}\right|_{R(-a)}$ is an isomorphism, we can cancel out $R(-a)$ and $\lambda_{0}(R(-a))$ from $\operatorname{con}\left(\mu_{\bullet}\right)_{0}$ and $\operatorname{con}\left(\mu_{\bullet}\right)_{-1}$ respectively, to obtain a complex $F_{\bullet}^{\prime}$ such that $F_{i}^{\prime}=\operatorname{con}\left(\mu_{\bullet}\right)_{i}$ for $i>0, F_{0}^{\prime}=\operatorname{con}\left(\mu_{\bullet}\right)_{0} / R(-a), F_{-1}^{\prime}=\operatorname{con}\left(\mu_{\bullet}\right)_{-1} / \lambda_{0}(R(-a))$ $\cong K_{0}(c), \operatorname{rank}_{R}\left(F_{-1}^{\prime}\right)=-1, H_{i}\left(\operatorname{con} \mu_{\bullet}\right) \cong H_{i}\left(F_{\bullet}^{\prime}\right)$ for all $i \in \mathbf{Z}$. Hence $F_{\bullet}^{\prime} \in$ $\mathcal{C}\left(p,-1, \mathfrak{a}^{\prime}\right)$ with $\mathfrak{a}^{\prime}:=\left(f_{1}, \ldots, f_{p}\right)$ as desired.

Lemma 2. Let $p \geq 2, s \leq-1, \mathfrak{a}$, and $F_{\bullet} \in \mathcal{C}(p, s, \mathfrak{a})$ be as in the previous lemma. In each of the three cases there, we may assume that the differential $\varphi_{\bullet}^{\prime}$ of $F_{\bullet}^{\prime}$ satisfies $\operatorname{Im}\left(\varphi_{p}^{\prime \vee}\right)=\operatorname{Im}\left(\varphi_{p}^{\vee}\right)$ and $\varphi_{i}^{\prime}=\varphi_{i}$ for $i>p$.

Proof. In each case, the differential $\varphi_{p}^{\prime}$, obtained by the method described in the proof above, is of the form

$$
\left(\begin{array}{c}
\varphi_{p} \\
0
\end{array}\right): F_{p} \longrightarrow F_{p-1} \oplus L
$$

with a graded free $R$-module $L$. Hence $\operatorname{Im}\left(\varphi_{p}^{\prime} \vee\right)=\operatorname{Im}\left(\varphi_{p}^{\vee}\right)$. Likewise $\varphi_{i}^{\prime}=\varphi_{i}$ for all $i>p$.

Theorem 3. Let $p \geq 2$ be an integer and let $M$ be a finitely generated torsionfree graded $R$-module with no free direct summand satisfying $\operatorname{Ext}_{R}^{i}(M, R)=0$ for $i=1, \ldots, p-1$. Then there exists a homogeneous ideal $I$ in $R$ of height $p$ which fits into an exact sequence of the form

$$
0 \longrightarrow S_{p-1} \longrightarrow \cdots \longrightarrow S_{1} \longrightarrow S_{0} \oplus M \longrightarrow I(c) \longrightarrow 0,
$$

where $c$ is an integer and $S_{i}(0 \leq i \leq p-1)$ are finitely generated graded free $R$-modules. 
Proof. If $M=0$, then any Cohen-Macaulay homogeneous ideal $I$ of height $p$ will do. Suppose $M \neq 0$. Let

$$
\cdots \stackrel{\varphi_{p+1}}{\longrightarrow} F_{p} \stackrel{\varphi_{p}}{\longrightarrow} F_{p-1} \stackrel{\varphi_{p-1}}{\longrightarrow} \cdots \stackrel{\varphi_{2}}{\longrightarrow} F_{1} \stackrel{\varphi_{1}}{\longrightarrow} F_{0} \longrightarrow M \longrightarrow 0
$$

be a minimal free resolution of $M$ over $R$. Let further

$$
\cdots \stackrel{\varphi_{-2}^{\vee}}{\longrightarrow} F_{-2}^{\vee} \stackrel{\varphi_{-1}^{\vee}}{\longrightarrow} F_{-1}^{\vee} \stackrel{\varphi_{0}^{\vee}}{\longrightarrow} F_{0}^{\vee} \stackrel{\varphi_{1}^{\vee}}{\longrightarrow} \operatorname{Im}\left(\varphi_{1}^{\vee}\right) \longrightarrow 0
$$

be a minimal free resolution of $\operatorname{Im}\left(\varphi_{1}^{\vee}\right)$ over $R$. Connecting the former to the dual of the latter, we obtain a complex

$$
F_{\bullet}: \cdots \stackrel{\varphi_{p+1}}{\longrightarrow} F_{p} \stackrel{\varphi_{p}}{\longrightarrow} F_{p-1} \stackrel{\varphi_{p-1}}{\longrightarrow} \cdots \stackrel{\varphi_{2}}{\longrightarrow} F_{1} \stackrel{\varphi_{1}}{\longrightarrow} F_{0} \stackrel{\varphi_{0}}{\longrightarrow} F_{-1} \stackrel{\varphi_{-1}}{\longrightarrow} F_{-2} \stackrel{\varphi_{-2}}{\longrightarrow} \cdots
$$

bounded on both sides (cf. [4]). Since $H_{i}\left(F_{\bullet}\right)=\operatorname{Ext}_{R}^{p-i}\left(\operatorname{Coker}\left(\varphi_{p}^{\vee}\right), R\right)$ for $i<p$, the codimension of $H_{0}\left(F_{\bullet}\right)$ in $\operatorname{Spec}(R)$ is larger than or equal to $p$. Besides,

$$
0 \longrightarrow H_{0}\left(F_{\bullet}\right) \longrightarrow M=F_{0} / \operatorname{Im}\left(\varphi_{1}\right) \longrightarrow F_{-1}
$$

is exact and $M$ is torsion-free. Hence $H_{0}\left(F_{\bullet}\right)=0$ and $\operatorname{rank}_{R}\left(F_{-1}\right)>0$. On the other hand, $\operatorname{Ext}_{R_{\mathfrak{p}}}^{i}\left(M_{\mathfrak{p}}, R_{\mathfrak{p}}\right)=0(1 \leq i \leq p-1)$ for all $\mathfrak{p} \in \operatorname{Spec}(R)$ with ht $(\mathfrak{p}) \leq p-1$ by hypothesis, so that there is a homogeneous ideal $\mathfrak{a}$ of height larger than or equal to $p$ such that $M_{\mathfrak{p}}$ is free if $\mathfrak{p} \in \operatorname{Spec}(R)$ and $\mathfrak{p} \not \supset \mathfrak{a}$. By our construction of the complex $F_{\bullet}$, this means that the localization $\left(F_{\bullet}\right)_{\mathfrak{p}}$ is split exact for all prime ideals $\mathfrak{p}$ not containing $\mathfrak{a}$. The support of $H_{i}\left(F_{\bullet}\right)$ is therefore contained in $\operatorname{Spec}(R / \mathfrak{a})$; in other words, $\mathfrak{a}^{l} H_{i}\left(F_{\bullet}\right)=0$ for all $i<0$ and $l \gg 0$. Thus $F_{\bullet} \in \mathcal{C}(p, s, \mathfrak{a})$ for some $s \leq-1$. Applying Lemmas 1 and 2 to $F_{\bullet}$, we can obtain a complex $G_{\bullet} \in$ $\mathcal{C}(p,-1, \mathfrak{b})$ such that $\operatorname{rank}_{R}\left(G_{-1}\right)=1, \operatorname{codim}\left(H_{-1}\left(G_{-1}\right)\right)=p, \operatorname{Im}\left(\psi_{p}^{\vee}\right)=\operatorname{Im}\left(\varphi_{p}^{\vee}\right)$, and $G_{i}=F_{i}, \psi_{i+1}=\varphi_{i+1}$ for $i>p-1$, where $\mathfrak{b}$ is a homogeneous ideal in $R$ of height larger than or equal to $p$ and $\psi_{\bullet}$ is the differential of $G_{\bullet}$. In fact, if $s<-1$,

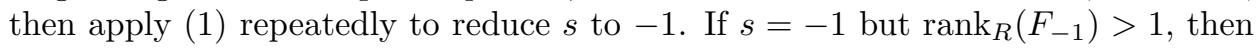
apply (2) repeatedly to reduce $\operatorname{rank}_{R}\left(F_{-1}\right)$ to one. If $s=-1$ and $\operatorname{rank}_{R}\left(F_{-1}\right)=1$ but $\operatorname{codim}\left(H_{-1}\left(F_{\bullet}\right)\right)>p$, then apply $(3)$ to reduce $\operatorname{codim}\left(H_{-1}\left(F_{\bullet}\right)\right)$ to $p$. For such a $G_{\bullet}$, let $c$ be the integer such that $G_{-1}=R(c)$ and $I \subset R$ the ideal of height $p$ such that $\operatorname{Im}\left(\psi_{0}\right)=I(c)$. Now, reverse the procedure. The complex

$$
\cdots \stackrel{\psi_{p+1}}{\longrightarrow} G_{p} \stackrel{\psi_{p}}{\longrightarrow} G_{p-1} \stackrel{\psi_{p-1}}{\longrightarrow} \cdots \stackrel{\psi_{1}}{\longrightarrow} G_{0} \stackrel{\psi_{0}}{\longrightarrow} G_{-1} \longrightarrow \operatorname{Coker}\left(\psi_{0}\right) \longrightarrow 0
$$

is a free resolution of $\operatorname{Coker}\left(\psi_{0}\right)=R / I(c)$, and

$$
\cdots \stackrel{\varphi_{-1}^{\vee}}{\longrightarrow} F_{-1}^{\vee} \stackrel{\varphi_{0}^{\vee}}{\longrightarrow} F_{0}^{\vee} \stackrel{\varphi_{1}^{\vee}}{\longrightarrow} \cdots \stackrel{\varphi_{p-1}^{\vee}}{\longrightarrow} F_{p-1}^{\vee} \stackrel{\varphi_{p}^{\vee}}{\longrightarrow} \operatorname{Im}\left(\varphi_{p}^{\vee}\right)=\operatorname{Im}\left(\psi_{p}^{\vee}\right) \longrightarrow 0
$$

is a free resolution of $\operatorname{Im}\left(\psi_{p}^{\vee}\right)$. Since $F_{i}=G_{i}$ for all $i>p-1$, our assertion follows from [6, Lemma 1.3].

Remark 4. One can give another proof of [10, Theorem 1.3] by an argument similar to that in the proof of (2) of Lemma 1.

\section{REFERENCES}

[1] M. Amasaki, On the structure of arithmetically Buchsbaum curves in $\mathbf{P}_{k}^{3}$, Publ. RIMS, Kyoto Univ. 20 (1984), 793 - 837. MR 86a:14027

[2] M. Amasaki, Integral arithmetically Buchsbaum curves in $\mathbf{P}^{3}$, J. Math. Soc. Japan 41, No. 1 (1989), 1 - 8. MR 90c: 14016

[3] M. Amasaki, Application of the generalized Weierstrass preparation theorem to the study of homogeneous ideals, Trans. AMS 317 (1990), 1 - 43. MR 90d:13002 
[4] M. Amasaki, Free complexes defining maximal quasi-Buchsbaum graded modules over polynomial rings, J. Math. Kyoto Univ. 33, No. 1 (1993), 143 - 170. MR 94a:13020

[5] M. Amasaki, Generators of graded modules associated with linear filter-regular sequences, J. Pure Appl. Algebra 114 (1996), 1 - 23. CMP 97:05

[6] M. Amasaki, Basic sequences of homogeneous ideals in polynomial rings, J. Algebra 190 (1997), 329 - 360. MR 98c:13029

[7] M. Amasaki, Basic sequence and Nollet's $\theta_{X}$ of a homogeneous ideal of height two, preprint (August, 1996).

[8] E. Ballico, G. Bolondi and J. C. Migliore, The Lazarsfeld-Rao problem for liaison classes of two-codimensional subschemes of $\mathbf{P}^{n}$, Amer. J. Math. 113 (1991), 117-128. MR 92c:14047

[9] N. Bourbaki, "Algèbre Commutative", Masson, Paris, 1985.

[10] A. V. Geramita and J. C. Migliore, A generalized liaison addition, J. Algebra 163 (1994), 139 - 164. MR 94m:14066

[11] M. Martin-Deschamps et D. Perrin, Sur la classification des courbes gauches, Astérisque 184 - 185, Société Mathématique de France, 1990. MR 91h:14039

[12] S. Nollet, Even linkage classes, Trans. AMS 348 (1996), 1137 - 1162. MR 96h:14069

[13] S. Nollet, Integral subschemes of codimension two, preprint.

[14] A. P. Rao, Liaison equivalence classes, Math. Ann. 258 (1981), 169 - 173. MR 83j:14045

Faculty of School Education, Hiroshima University, 1-1-1 Kagamiyama, Higashi-HiroSHIMA 739-8524, JAPAN

E-mail address: amasaki@ipc.hiroshima-u.ac.jp 